\title{
The Ideal Stimulation Protocol: Is There One?
}

\author{
Gautam N. Allahbadia ${ }^{1,2}$
}

Published online: 1 July 2015

(C) Federation of Obstetric \& Gynecological Societies of India 2015

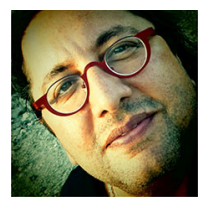

\begin{abstract}
About the Author
Gautam N. Allahbadia is the Editor-in-chief of the Journal of Obstetrics \& Gynecology of India as well as the IVF Lite (Journal of Minimal Stimulation IVF). He is the Medical Director of Rotunda-The Center For Human Reproduction, the world-renowned fertility clinic at Bandra, Mumbai, India - as well as the New Hope IVF Clinic at Sharjah, UAE. He is a noted world authority on. Ultrasound-guided Embryo Transfers, and one of the pioneers in Third Party Reproduction in South-East Asia. Dr. Allahbadia was responsible for India's first trans-ethnic surrogate pregnancy involving a Chinese couple's baby delivered by an unrelated Indian surrogate mother. He has over 100 peer-reviewed publications to his credit and is on the Editorial Board of several International Journals. Throughout his career, Dr. Allahbadia has been instrumental in developing new fertility-enhancing protocols and propagating the use of Ultrasound in Embryo Transfer procedures. You
\end{abstract} can read more about his work at www.gautamallahbadia.com

\section{Introduction}

The first successful in vitro fertilization (IVF) attempt and most treatment cycles for a while thereafter were conducted in spontaneous menstrual cycles. Nevertheless, realization that availability of a crop of mature oocytes markedly increased chances of success in this therapy prompted most centers to adopt some form of controlled

Gautam N. Allahbadia is the Medical Director at Rotunda-The Center For Human Reproduction, Mumbai, India \& New Hope IVF, Sharjah, UAE.

Gautam N. Allahbadia

ivfwaladoc@gmail.com

1 Rotunda-The Center For Human Reproduction, Mumbai, India

2 New Hope IVF, Sharjah, UAE ovarian hyperstimulation $(\mathrm{COH})$. At the outset, clomiphene citrate alone or in combination with human menopausal gonadotropins (hMG) was used, but eventually exogenous gonadotropins emerged as the sole stimulatory drug for $\mathrm{COH}$. Exogenous gonadotropins, and specifically hMG products, have been used in the treatment of infertility since the 1960s, when the first hMG product became available. Subsequently, over the last 25 years, they have become the mainstay of fertility treatment worldwide. The gonadotropins are indicated in isolation as a treatment to induce ovulation, normally in cases where Clomiphene has failed or as a first-line treatment in specific cases of amenorrhea. They are also indicated for Hypogonadotropic hypogonadism in men and women. The most widespread use of gonadotropins is for women undergoing superovulation within a medically assisted reproductive program, like IVF. Superovulation is the stimulation of the ovaries to produce more than one follicle, which enables several embryos to be created. We are still in the search for that 
"perfect" or "ideal" ovarian stimulation protocol combining both GnRH analogs and gonadotropins that will give us an "adequate" number of oocytes; these oocytes should be of good quality resulting into embryos with a very good morphokinetic score [1] with a high implantation potential and with elimination of ovarian hyperstimulation syndrome (OHSS). The resulting pregnancies should carry to term as a result of the optimal uterine and endocrinological environment that has resulted as a consequence of using that "ideal" stimulation protocol. In the quest for that perfect stimulation protocol, we must imbibe knowledge of the use of GnRH analogs and gonadotropins in different endocrine milieus such as "poor responders" and from natural and artificially stimulated hypogonadotropic hypogonadic states. Newer Gonadotropin preparations are being introduced continuously over the past few years in the quest of that "perfect" but elusive stimulation protocol. Research and practice in the field has led to the era of "Individualized" treatment protocols in the last decade [2, 3].

\section{Discussion}

The main objective of individualization of stimulation protocols in IVF is to offer every single woman the best protocol tailored to her own unique characteristics, thus maximizing the chances of pregnancy and eliminating the iatrogenic and avoidable risks resulting from ovarian stimulation. Personalization of treatment in IVF should be based on the prediction of ovarian response for every individual. The starting point is to identify if a woman is likely to have a normal, poor, or a hyper response and choose the ideal treatment protocol tailored to this prediction.

Human menopausal gonadotropins were initially used for $\mathrm{COH}$, but poor reproductive outcome was ascribed to high levels of circulating LH associated with hMG therapy [4]. Lunenfeld hypothesized "that a high concentration of LH through the follicular phase allows the developing Oocyte to mature prematurely, producing at ovulation an Oocyte that is physiologically aged. Such Oocytes may have a decreased capacity to fertilize; if they fertilize, they are unlikely to implant; and if they implant, their survival rate is decreased, resulting in early abortion" [4]. The LH hypothesis changed worldwide thinking and the direction of stimulation protocols in ART. "Pure" FSH preparations with reduced LH content such as Purified FSH (PoFSH) [5], Highly Purified FSH (PoFSH-HP) [6], Recombinant FSH (recFSH) [7], and Biosimilar recFSH [8] were introduced over the last three decades into clinical practice. There was no consensus reached on the superiority of one preparation over the other till date [9-11].

Soon after the introduction of $\mathrm{COH}$ in ART, it became evident that in the course of ovulation induction, the midcycle LH surge could be unexpectedly triggered by rising ovarian steroid levels; as a result, premature ovulation or follicle luteinization could occur and cause cycle cancelations in up to $30 \%$ of cases [12]. Thus, in the mid-1980s, GnRH agonist supplementation was tested in ovulation induction and then successfully applied in most cycles [13]. The major advantage in the use of GnRH agonist is the complete elimination of the preovulatory LH surge; in addition to preventing cycle cancelations due to premature ovulation, the use of GnRH agonist has permitted to dramatically reduce endocrine monitoring and its related costs [14]. Next, GnRH antagonists were introduced in stimulation and offered immediate yet completely reversible competitive blockade of the $\mathrm{GnRH}$ receptors at the level of the pituitary [15]. The use of GnRH analogs in conjunction with gonadotropins for $\mathrm{COH}$ has afforded better control of the cycle, and has provided versatility to individualize specific $\mathrm{COH}$ protocols to specific groups of patients.

Marca and Sunkara published a systematic review of the existing literature by searching Medline, EMBASE, Cochrane library, and Web of Science for publications in the English language related to $\mathrm{AFC}, \mathrm{AMH}$, and their incorporation into controlled ovarian stimulation (COS) protocols in IVF [16]. The literature review demonstrated that antral follicle count (AFC) and anti-mullerian hormone $(\mathrm{AMH})$, the most sensitive markers of ovarian reserve identified to date, are ideal in planning personalized $\mathrm{COH}$ protocols. These sensitive markers permit prediction of the whole spectrum of ovarian response with reliable accuracy, and clinicians may use either of the two markers as they can be considered interchangeable [16].

Rinaldi et al. published a prospective, open, randomized study which included 349 infertile patients considered at high risk of developing OHSS, undergoing IVF treatment in two private, assisted reproduction centers [17]. The patients were randomized into two groups: group A ( $n=148)$ had a mild/minimal stimulation protocol of recombinant FSH (rFSH) combined with GnRH antagonist. Group B ( $n=201)$ (control group) had a standard long protocol of $\mathrm{rFSH}$ combined with GnRH agonist. There were no significant differences observed between the two groups regarding the mean number of oocytes retrieved per patient, mature metaphase II oocytes, fertilization rate, and embryo cleavage rate. Significantly higher implantation rate $(21.5$ vs. $14.5 \%)(p<0.05)$, pregnancy rate $(37.7$ vs. $23.4 \%)(p<0.05)$, and delivery rate $(32.8$ vs. $20.1 \%)$ $(p<0.05)$ were observed in favor of groups A compared to group B. Lower proportion of patients (4.7\%), although not statistically significant, has developed OHSS in group A compared to group B $(8.4 \%)$. Their study shows that mild stimulation regimen is highly effective for ovarian stimulation of patients who have experienced OHSS complication without increasing the risk of OHSS [17]. 
Natural cycle [18], modified natural cycle [19], and IVF Lite [20] are different protocols, which provide some advantages compared to conventional stimulation: lower medication cost, less injections, less invasive, low risk of ovarian hyperstimulation, and multiple pregnancy [18-20]. Their main drawback is high cancelation rate due to premature ovulation and lack of egg recovery at the retrieval. When an embryo transfer can be performed, the cumulative pregnancy rate is similar to the results in conventional stimulation [18-20]. These protocols do not presently provide any advantage in terms of results for woman with normal ovarian reserve; however, they must be considered as a very good therapeutic alternative in poor responders.

Contemporary research advocates the elective use of the GnRH antagonist-based regimen for hyper-responders [21], and probably also for poor responders [22], as likely to be beneficial. The incidence of poor ovarian responders among infertile women has been estimated at 9-24\%, but according to recent reviews, it seems to have slightly increased [23]. Current literature proposes new risk factors which could be the cause of a reduction in ovarian reserve, which also includes genetic factors. The use of the "Bologna criteria" and the introduction of long-acting gonadotropin in clinical practice have given rise to new promising stimulation protocols for this group of patients [24]. Delayed-start antagonist protocol (estrogen priming followed by early follicular-phase GnRH antagonist treatment for 7 days before ovarian stimulation) improves ovarian response in poor responders by promoting and synchronizing follicle development without impairing the oocyte developmental competence [24].

Controlled ovarian stimulation directly influences assisted reproductive technology (ART) outcomes. Indeed, several studies have shown that the total IU of gonadotropins used for ovarian stimulation inversely correlates with pregnancy rate. Nowadays, two main gonadotropins are used in ART protocols: human-derived and recombinant follicle-stimulating hormone (FSH). The difference between these two hormones is dramatic. Indeed, the human-derived FSH is an acidic isoform of the hormone, while the recombinant is a less-acidic one [25]. In particular, during a physiological menstrual cycle, the acidic isoform is produced during the follicular phase (probably it is more effective in recruiting follicles), while less-acidic isoform is produced during the mid-follicular phase (preovulatory). In a recent study, Gerli et al. evaluated the efficacy of a protocol that mimics the physiological shift from an acidic to a less-acidic FSH isoform during oocyte maturation [25]. Patients were randomized in two groups: group 1, patients that received $225 \mathrm{IU}$ of human-derived FSH (hFSH Fostimon, IBSA, Lodi, Italy) for 6 days from the second day of the cycle, followed by $225 \mathrm{IU}$ of recombinant FSH (rFSH Gonal-F; Serono, Rome, Italy) from the 7th day of stimulation until hCG administration; and group 2, control group, patients that received 225 IU recombinant FSH alone from the second day of the cycle until hCG administration. The combined protocol $(\mathrm{hFSH}+\mathrm{rFSH})$ resulted in significantly less IU of FSH necessary for ovarian stimulation together with the stimulation days. Furthermore, oocyte and embryo quality was higher in the group of patients treated with the combined protocol. Noteworthy, a significantly higher implantation rate and pregnancy rate were observed in favor of group 1 compared to group 2 [25]. Rinaldi et al. were able to supposedly establish a stimulation protocol mimicking the physiological differences in FSH isoforms, hFSH combined with $\mathrm{rFSH}$ to positively impact ART outcome [25].

Bemfola (follitropin alfa) (Finox AG, Switzerland), a new Biosimilar recombinant FSH, has a comparable pharmacological profile to that of Gonal-F (Merck Serono, Germany), the current standard for ovarian stimulation. A randomized, multicenter, Phase 3 study in women undergoing IVF or intracytoplasmic sperm injection $(n=372)$ showed Bemfola yielding similar efficacy and safety profiles to Gonal-F [8]. No difference in severe OHSS was observed between treatment groups (Bemfola: $0.8 \%$; Gonal-f: $0.8 \%$ ). This study demonstrated similar clinical efficacy and safety profiles between Bemfola and Gonal-F, and suggests that Biosimilars can be an appropriate alternative in ovarian stimulation protocols [8].

Since the introduction of the gonadotropin-releasing hormone analogs (GnRHa) protocol, it has become possible to trigger final oocyte maturation with a bolus of GnRHa [26]. This leads to a significant reduction or complete elimination of OHSS compared with human chorionic gonadotrophin (hCG) trigger [26]. Early trials showed a severe luteal-phase insufficiency after GnRHa trigger, despite the application of standard luteal-phase support protocols [27]. Subsequent research has led to modifications of the luteal-phase support, resulting in reproductive outcome comparable to that seen after hCG trigger in normal and high responders $(\mathrm{HH})$. GnRHa trigger facilitates a tailored approach to subsequent luteal-phase support, taking into account the ovarian response to stimulation. In the future, GnRHa is likely to be used for trigger in all women co-treated with GnRH antagonists [28].

In vitro fertilization cycles generate abnormalities in luteal-phase sex steroid concentrations, and this represents an important limiting factor to achieve a good pregnancy rate. Although there are evidences about the usefulness of luteal-phase support (LPS) after IVF cycles, no consensus exists about the best dose and the way of progesterone (PG) administration, the advantages of estradiol (E2) supplementation, and which IVF protocol could benefit from one more than other LPS schemes [29]. The aim of a 2014 study was to assess the best LPS (low-dose PG, high-dose 
PG, high-dose PG, and E2 supplementation) to achieve the highest clinical/ongoing pregnancy rate [30, 31]. They set up a randomized trial on 360 women undergoing IVF (180 treated by long-GnRH agonist, 90 by short-GnRH agonist, and 90 by short-GnRH antagonist protocol) and stimulated by recombinant FSH alone. Their data demonstrated that high-dose PG is better than low-dose one to increase both clinical and ongoing pregnancy rates [30, 31].

In the early follicular phase, a flare-up effect of gonadotropin releasing hormone $(\mathrm{GnRH})$ agonists and incomplete luteolysis in $\mathrm{GnRH}$ antagonist regimens can result in significant elevations of progesterone. In the late follicular phase, progesterone elevations in GnRH analog cycles are the result of the ovarian stimulation itself, driven by high follicle stimulating hormone dosage, estradiol levels, and the numbers of follicles and oocytes. It seems that progesterone elevations ( $\geq 1.5 \mathrm{ng} / \mathrm{mL}$ or $4.77 \mathrm{nmol} / \mathrm{L}$ ) have a detrimental effect on the outcome of pregnancy, accelerating the endometrial maturation [32, 33]. The most appropriate choice to avoid the negative effects of follicular progesterone elevations is to cancel fresh embryo transfer and to transfer frozen-thawed embryos in natural cycles. To prevent follicular-phase elevations, it might be preferable to use milder stimulation protocols, earlier trigger of ovulation in high responders, and single-blastocyst transfer on day 5 [32]. The optimal GnRH analog protocols during the entire stimulation period appear to be the long agonist as well as "long" and long GnRH antagonist regimens [33].

\section{Recent Advances}

In Kuang et al.'s study group, hMG and Medroxyprogesterone acetate (MPA) were administered simultaneously beginning on cycle day 3 [34]. Ovulation was induced with a GnRH agonist or co-triggered by a GnRH agonist and hCG when dominant follicles matured. A short protocol was used in the control group. Viable embryos were cryopreserved for later transfer in both protocols. In their study group, LH suppression persisted during ovarian stimulation, and the incidence of premature LH surge was $0.7 \%$ (1/150). No statistically significant differences were found in the clinical pregnancy rates (47.8 vs. $43.3 \%$ ), implantation rates (31.9 vs. $27.7 \%)$, and live-birth rates (42.6 vs. $35.5 \%$ ) in both the study group and controls. These results show that MPA is an effective oral alternative for the prevention of premature LH surge in woman undergoing $\mathrm{COH}$ [34]. This finding will help establish a new "individualized" regimen for ovarian stimulation in combination with embryo cryopreservation.

\section{Conclusion}

These individualized stimulation protocols offer several benefits; it enables clinicians to give women more accurate information on their prognosis thus facilitating counseling especially in cases of extremes of ovarian response. The deployment of various therapeutic options based on individualized use of $\mathrm{GnRH}$ analogs and the fine tuning of the gonadotropin dose on the basis of potential ovarian response in IVF patients of different ages will make IVF more effective and safer in years to come. New parenteral, transdermal, inhaled agents and oral fertility drugs, and protocols are currently being researched to further simplify treatment for ART. However, the moot question of whether there is an ideal stimulation protocol in the year 2015 remains a "work in progress."

Conflict of interest None.

\section{References}

1. Basile N, Vime P, Florensa M, et al. The use of morphokinetics as a predictor of implantation: a multicentric study to define and validate an algorithm for embryo selection. Hum Reprod. 2015;30(2):276-83. doi:10.1093/humrep/deu331 (Epub 2014 Dec 19. PubMed PMID: 25527613).

2. Nardo LG, Fleming R, Howles CM, et al. Conventional ovarian stimulation no longer exists: welcome to the age of individualized ovarian stimulation. Reprod Biomed Online. 2011;23(2):141-8. doi:10.1016/j.rbmo.2011.05.008 (Epub 2011 May 19).

3. Bosch E, Ezcurra D. Individualised controlled ovarian stimulation (iCOS): maximising success rates for assisted reproductive technology patients. Reprod Biol Endocrinol. 2011;21(9):82. doi: 10.1186/1477-7827-9-82.

4. Lunenfeld B, Lunenfeld E. Gonadotropic preparations-lessons learnt. Fertil Steril. 1997;67(5):812-4.

5. Fleming R, Lloyd F, Herbert M, et al. Effects of profound suppression of LH during controlled ovarian stimulation on follicular activity, oocyte and embryo function in cycles stimulated with purified FSH. Hum Reprod. 1988;13(9):1788-92.

6. Westergaard LG, Erb K, Laursen S. The effect of human menopausal gonadotropin and highly purified, urine-derived follicle stimulating hormone on the outcome of in vitro fertilization in down-regulated normogonadotropic women. Hum Reprod. 1996;11:1209-13.

7. Jacob S, Drudy L, Conroy R, et al. Outcome from consecutive in vitro fertilization/intracytoplasmic sperm injection attempts in the final group treated with urinary gonadotrophins and the first group treated with recombinant follicle stimulating hormone. Hum Reprod. 1998;13(7):1783-7.

8. Rettenbacher M, Andersen AN, Garcia-Velasco JA, et al. A multi-centre phase 3 study comparing efficacy and safety of Bemfola $\left({ }^{\circledR}\right)$ versus Gonal-f $\left({ }^{\circledR}\right)$ in women undergoing ovarian stimulation for IVF. Reprod Biomed Online. 2015;30(5):504-13. doi:10.1016/j.rbmo.2015.01.005 (Epub 2015 Jan 27).

9. Check JH, Slovis B. Choosing the right stimulation protocol for in vitro fertilization-embryo transfer in poor, normal, and hyperresponders. Clin Exp Obstet Gynecol. 2011;38(4):313-7. 
10. Jansen CA, van Os $\mathrm{HC}$, Out $\mathrm{HJ}$, et al. A prospective randomized clinical trial comparing recombinant follicle stimulating hormone (Puregon) and human menopausal gonadotrophins (Humegon) in non-down-regulated in vitro fertilization patients. Hum Reprod. 1998;13(11):2995-9.

11. van Tilborg TC, Eijkemans MJ, Laven JS, et al. The OPTIMIST study: optimisation of cost effectiveness through individualised FSH stimulation dosages for IVF treatment. A randomised controlled trial. BMC Womens Health. 2012;12:29.

12. Cedars MI, Surey E, Hamilton E, et al. Leuprolide acetate lowers circulating, bioactive luteinizing hormone and testosterone concentrations during ovarian stimulation for oocyte retrieval. Fertil Steril. 1990;53:627-31.

13. Hughes FG, Fedorkow DM, Daya S, et al. The routine use of gonadotropin releasing hormone agonists prior to in vitro fertilization and gamete intrafallopian tube of randomized controlled. Fertil Steril. 1992;58:888-90.

14. Meldrum D. GnRH agonists as adjuncts for in vitro fertilization. Obstet Gynecol Surv. 1989;44:314-7.

15. Marci R, Graziano A, Lo Monte G, et al. GnRH antagonists in assisted reproductive techniques: a review on the Italian experience. Eur Rev Med Pharmacol Sci. 2013;17(7):853-73.

16. La Marca A, Sunkara SK. Individualization of controlled ovarian stimulation in IVF using ovarian reserve markers: from theory to practice. Hum Reprod Update. 2014;20(1):124-40. doi: 10.1093/humupd/dmt037 (Epub 2013 Sep 29).

17. Rinaldi L, Lisi F, Selman H. Mild/minimal stimulation protocol for ovarian stimulation of patients at high risk of developing ovarian hyperstimulation syndrome. $\mathrm{J}$ Endocrinol Invest. 2014;37(1):65-70. doi:10.1007/s40618-013-0021-1 (Epub 2014 Jan 8).

18. Bodri D, Kawachiya S, Kondo M, et al. Oocyte retrieval timing based on spontaneous luteinizing hormone surge during natural cycle in vitro fertilization treatment. Fertil Steril. 2014;101(4): 1001-7.e2. doi:10.1016/j.fertnstert.2014.01.016 (Epub 2014 Feb 15. PubMed PMID: 24534290).

19. von Wolff M, Rohner S, Santi A, et al. Modified natural cycle in vitro fertilization an alternative in vitro fertilization treatment with lower costs per achieved pregnancy but longer treatment time. J Reprod Med. 2014;59(11-12):553-9 PubMed PMID: 25552127.

20. Gandhi GN, Allahbadia GN, Kagalwala S, et al. IVF lite-a new strategy for managing poor ovarian responders. IVF Lite. 2014;1:22-8.

21. Lin $\mathrm{H}, \mathrm{Li} \mathrm{Y}$, Li L, et al. Is a GnRH antagonist protocol better in PCOS patients? A meta-analysis of RCTs. PLoS ONE. 2014;9(3):e91796. doi:10.1371/journal.pone.0091796 (eCollection 2014).

22. Sunkara SK, Coomarasamy A, Faris R, et al. Long gonadotropinreleasing hormone agonist versus short agonist versus antagonist regimens in poor responders undergoing in vitro fertilization: a randomized controlled trial. Fertil Steril. 2014;101(1):147-53. doi:10.1016/j.fertnstert.2013.09.035 (Epub 2013 Nov 1).
23. Ubaldi F, Vaiarelli A, D'Anna R, et al. Management of poor responders in IVF: is there anything new? Biomed Res Int. 2014;2014:352098. doi:10.1155/2014/352098 (Epub 2014 Jul 20).

24. Cakmak H, Tran ND, Zamah AM, et al. A novel "delayed start" protocol with gonadotropin-releasing hormone antagonist improves outcomes in poor responders. Fertil Steril. 2014;101(5): 1308-14. doi:10.1016/j.fertnstert.2014.01.050 (Epub 2014 Mar 14).

25. Gerli S, Di Renzo GC. Establishing a combined stimulation protocol hFSH followed by rFSH might represent a breakthrough in the IVF practice. Eur Rev Med Pharmacol Sci. 2013;17(15): 2091-6.

26. Humaidan P, Alsbjerg B. GnRHa trigger for final oocyte maturation: is HCG trigger history? Reprod Biomed Online. 2014;29(3):274-80. doi:10.1016/j.rbmo.2014.05.008 (Epub 2014 Jun 12).

27. Fatemi HM, Polyzos NP, van Vaerenbergh I, et al. Early luteal phase endocrine profile is affected by the mode of triggering final oocyte maturation and the luteal phase support used in recombinant follicle-stimulating hormone-gonadotropin-releasing hormone antagonist in vitro fertilization cycles. Fertil Steril. 2013;100(3):742-7. doi:10.1016/j.fertnstert.2013.05.028 (Epub 2013 Jun 24).

28. Yding Andersen C, Vilbour Andersen K. Improving the luteal phase after ovarian stimulation: reviewing new options. Reprod Biomed Online. 2014;28(5):552-9. doi:10.1016/j.rbmo. 2014.01.012 (Epub 2014 Feb 5. Review).

29. Vaisbuch E, de Ziegler D, Leong M, et al. Luteal-phase support in assisted reproduction treatment: real-life practices reported worldwide by an updated website-based survey. Reprod Biomed Online. 2014;28(3):330-5. doi:10.1016/j.rbmo.2013.10.022 (Epub 2013 Nov 14).

30. Gizzo S, Andrisani A, Esposito F, et al. Which luteal phase support is better for each IVF stimulation protocol to achieve the highest pregnancy rate? A superiority randomized clinical trial. Gynecol Endocrinol. 2014;30:1-7 (Epub ahead of print).

31. Howles CM. Role of LH and FSH in ovarian function. Mol Cell Endocrinol. 2000;161(1-2):25-30.

32. Kasum M, Simunić V, Vrčić H, et al. Follicular progesterone elevations with ovulation induction for IVF. Gynecol Endocrinol. 2014;30(8):537-41. doi:10.3109/09513590.2014.916263 (Epub 2014 May 19).

33. Kasum M, Radakovic B, Simunic V, et al. Preovulatory progesterone rise during ovarian stimulation for IVF. Gynecol Endocrinol. 2013;29(8):744-8. doi:10.3109/09513590.2013.798280 (Epub 2013 Jun 7).

34. Kuang Y, Chen Q, Fu Y, et al. Medroxyprogesterone acetate is an effective oral alternative for preventing premature luteinizing hormone surges in women undergoing controlled ovarian hyperstimulation for in vitro fertilization. Fertil Steril. 2015;. doi: 10.1016/j.fertnstert.2015.03.022 (Epub ahead of print). 